\title{
Probing the Nuclear Neutron Skin by Low-Energy Dipole Modes
}

\author{
N. Tsoneva* and H. Lenske \\ Institut für Theoretische Physik, Universität Gießen, \\ Heinrich-Buff-Ring 16, D-35392 Gießen, Germany \\ Ch. Stoyanov \\ Institute for Nuclear Research and Nuclear Energy, 1784 Sofia, Bulgaria
}

(Dated: November 23, 2018)

\begin{abstract}
Dipole excitations below the neutron threshold in neutron rich Sn isotopes are studied theoretically in the Quasiparticle-Phonon Model with Hartree-Fock-Bogoliubov single particle input. Of special interest are the low-lying two-phonon $1^{-}$states and the Pygmy Dipole Resonance (PDR). The evolution of low-energy dipole excitations with neutron excess is investigated over the Sn isotopic chain including the experimentally unknown regions close to ${ }^{132} \mathrm{Sn}$. A dependence of the PDR strengths and centroid energies on the neutron skin thickness is found. Despite significant multiphonon contributions to mean energies and transition strengths, the PDR states retain their onephonon character. The fragmentation pattern is reduced with increasing neutron excess towards the $\mathrm{N}=82$ shell closure which will be of advantage for future experimental work.

PACS numbers: 21.60.Jz, 24.30.Cz, 27.60.+j
\end{abstract}

A genuine feature of neutron-rich nuclei is the appearance of low-energy electric dipole strength, seen recently in high-precision photon scattering experiments already in stable nuclei with small [1, 2] and moderate [3] neutron excess. These so-called Pygmy Dipole Resonances $(P D R)$ are observed as a clustering of states close to the neutron threshold which in presently accessible stable medium- and heavy-mass nuclei is at excitation energies $\mathrm{E}_{x} \sim 5.5-8 \mathrm{MeV}$. In a first attempt for experimental investigations on the dipole strength distribution in exotic nuclei with radioactive beams recently performed in MSU and GSI the neutron-rich (stable and unstable) oxygen isotopes are studied. A dipole strength is observed below [4] and above [5] the neutron threshold. The measurements of the latest in the energy region $5-8 \mathrm{MeV}$ for ${ }^{18,20} \mathrm{O}$ 'are consistent with that for other nuclei that may exhibit the Pygmy Dipole Resonance'. Although carrying only a small fraction of the full dipole strength the PDR states are of particular interest because they are expected to reflect the motion of the neutron skin against the core of normal nuclear matter. In order to obtain more direct evidence for such a peculiar mode studies of low-energy dipole excitations over sufficiently long isotopic chains are necessary. Here, we present results of an exploratory theoretical investigation for the neutron-rich Sn isotopes in experimentally less or even unknown regions at neutron numbers $\mathrm{N}=70-82$. As discussed in [3], the nature of the low-energy dipole strength differs significantly from the isovector Giant Dipole Resonance (GDR) mode where proton and neutron fluids as a whole move against each other. Moreover, the PDR mode has to be distinguished from the other known low-energy

*Also at Institute for Nuclear Research and $\mathrm{Nu}$ clear Energy, 1784 Sofia, Bulgaria; Electronic address: Nadia.Tsoneva@theo.physik.uni-giessen.de isoscalar dipole excitation, namely the two-phonon $1^{-}$ states resulting from the anharmonic interactions of the lowest $2^{+}$and $3^{-}$states in a nucleus. The anharmonicities are reflecting the intrinsic fermionic structure of the nuclear phonons thus deviating from ideal bosons. These competing effects are taken into account in an appropriate way by the Quasiparticle Phonon Model (QPM) [6]. Applications of the QPM to low-energy dipole strength [7, 8, 9, 10] and also in the recent PDR investigations in ${ }^{208} \mathrm{~Pb}$ [3] have led to very good descriptions of data thus giving confidence on the reliability of the model for such investigations.

The QPM approach is at present the only method allowing for sufficiently large configuration space such that a unified description of low-energy single and multiple phonon states and the GDR is feasible. In the onephonon sector the Quasiparticle Random-Phase Approximation (QRPA) is recovered as a well understood limiting case if the quasi-boson approximation is used. Such a unified treatment is exactly what is required in order to separate the multi-phonon and the genuine PDR $1^{-}$ strengths in a meaningful way. For the aim of this paper we use the standard form of QPM [6], i.e. approximating residual interactions in terms of separable multipolemultipole interactions with empirical coupling constants, see e.g. 11, 12. The isoscalar quadrupole and octupole coupling constants are chosen to reproduce the experimental energies and electromagnetic transitions of the $2_{1}^{+}$and $3_{1}^{-}$states. Since for $\mathrm{A}<126$ where the data are available the coupling constants vary smoothly with neutron number on a level of less than $5 \%$ extrapolations into the unexplored mass region towards ${ }^{132} \mathrm{Sn}$ can be done safely.

Since single particle energies and a reliable description of ground state properties in general are critical quantities for extrapolations of QRPA and QPM calculations into unknown mass regions here we put special emphasis 
on the mean-field part. Because of the numerical constraints set by the QPM a semi-microscopic approach is chosen. Taking advantage of the Kohn-Sham theorem [13] of Density Functional Theory (DFT) the total binding energy $B(A)$ is expressed as an integral over an energy density functional with (quantal) kinetic $(\tau)$ and self-energy parts, respectively,

$$
\begin{gathered}
B(A)=\int d^{3} r\left(\tau(\rho)+\frac{1}{2} \rho U(\rho)\right)+E_{\text {pair }} \\
=\sum_{j} v_{j}^{2}\left(e_{j}-<\Sigma>_{j}+\frac{1}{2}<U>_{j}\right)+E_{\text {pair }},
\end{gathered}
$$

and pairing contributions are indicated by $E_{\text {pair }}$. The second relation is obtained from Hartree-FockBogoliubov (HFB) theory with occupancies $v_{j}^{2}$ and potential energies $\langle U\rangle_{j}$ of the occupied levels $j$, see e.g. 14, 15. Above, $U(\rho)$ is the proper self-energy, i.e. not including the rearrangement contributions from the intrinsic density dependence of nuclear interactions [15, 16]. Hence, $U(\rho)$ has to be distinguished from the effective self-energy obtained by variation

$$
\Sigma(\rho)=\frac{1}{2} \frac{\partial \rho U(\rho)}{\partial \rho}
$$

and appearing in the single particle Schroedinger equation. In order to keep the QPM calculations feasible we choose $\Sigma \equiv U_{W S}$ to be of Wood-Saxon (WS) shape with adjustable parameters. By inversion and observing that the densities and potentials in a finite nucleus are naturally given parametrically as functions of the radius $r$, we find

$$
\rho(r) U(r)=-2 \int_{r}^{\infty} d s \frac{\partial \rho(s)}{\partial s} U_{W S}(s)
$$

Evaluating these relations with the microscopic proton and neutron densities obtained by solving the Schroedinger equation with $U_{W S}$ the potential $U(\rho)$ is the self-consistently derived reduced self-energy entering e.g. into the binding energy.

In practice, for a given nucleus of mass $A$ the depth of the central and spin-orbit potentials, radius and diffusivity parameters of $U_{W S}$ are adjusted separately for protons and neutrons to the corresponding single particle separation energies, the total binding energy [17, the charge radii and (relative) differences of proton and neutron root-mean-square (RMS) radii,

$$
\delta r=\sqrt{<r^{2}>_{n}}-\sqrt{<r^{2}>_{p}}
$$

from our previous HFB calculations [15, 16]. The theoretically obtained RMS radii are compared to those determined from charge exchange reactions by Krasznahorkay et al. [18, 19] for a number of Sn isotopes. However, since the measurements did not provide absolute $\delta r$ values the data were normalized in [18, 19] to theoretical

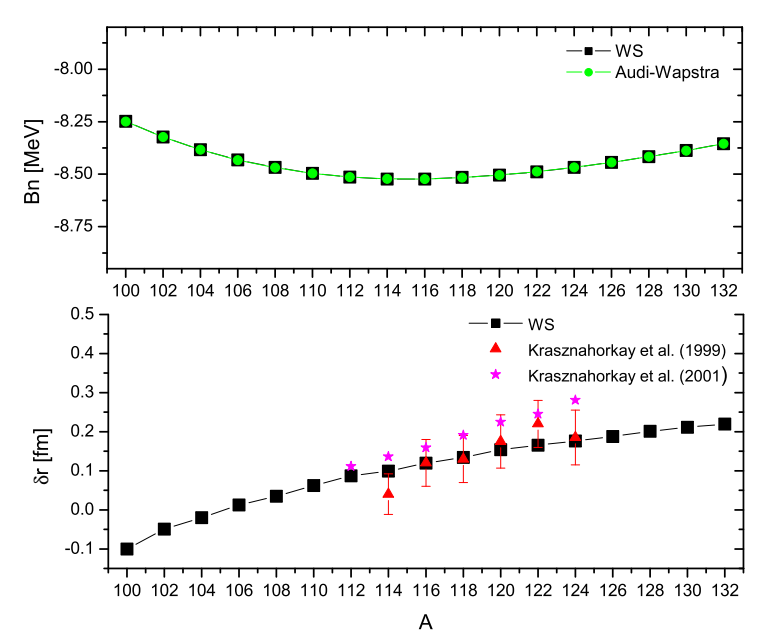

FIG. 1: Ground state properties of the Sn isotopes. In the upper panel the total nuclear binding energies per particle calculated with the adjusted WS potentials are compared to the values from the Audi-Wapstra compilation [17]. In the lower panel the calculated with the adjusted WS potentials differences of proton and neutron rms radii are compared to the experimental values obtained by Krasznahorkay et al. from charge exchange reactions in ref. [18, 19].

predictions. Hence, strictly spoken only the relative mass dependence of the experimental results shown in Fig [ is of significance. The HFB energies were scaled by an average effective mass of $m^{*} / m=0.68$ thus removing the known problem of unrealistically large HFB level spacings at the Fermi surface. The approach sketched above leads to very satisfactory results on binding energies and proton-neutron RMS-differences as shown in Fig पfor the Sn isotopes. A smooth dependence of the parameters on $A$ is found which supports the reliability of the method.

In the QPM calculations the structure of the excited states is described by a wave function including up to three-phonon configurations [8] which are built from a basis of QRPA states of natural parity excitations with $\mathrm{J}^{\pi}=1^{-} \div 5^{-}$. Since the one-phonon configurations up to $\mathrm{E}_{x}=20 \mathrm{MeV}$ are considered the core polarization contributions to the transitions of the low-lying $1^{-}$states are taken into account explicitly. Hence, we do not need to introduce effective charges. The two- and threephonon configurations are truncated at $\mathrm{E}_{x}=4.5 \mathrm{MeV}$ for the calculation of the quadrupole-octupole $1^{-}$state for the proper comparison with the available Nuclear Resonance Fluorescence (NRF) data [7]. For the QPM calculations between $4.5 \div 8 \mathrm{MeV}$ the two- and three-phonon basis is limited to states up to $\mathrm{E}_{x}=8.5 \mathrm{MeV}$ and $8 \mathrm{MeV}$, respectively.

As an important prerequisite for the reliability of the model wave functions we consider first the lowest $\mathrm{J}^{\pi}=2^{+}$ and $\mathrm{J}^{\pi}=3^{-}$states in the ${ }^{120 \div 130} \mathrm{Sn}$ isotopes. The QPM 
TABLE I: QPM results for the energies and the reduced $\mathrm{B}(\mathrm{E} 1), \mathrm{B}(\mathrm{E} 2)$ and $\mathrm{B}(\mathrm{E} 3)$ transition probabilities of the first $1^{-}, 2^{+}$and $3^{-}$states in ${ }^{120 \div 130} \mathrm{Sn}$ isotopes. A comparison with the experimental data [7] is presented.

\begin{tabular}{|c|c|c|c|c|c|c|}
\hline \multirow[t]{2}{*}{ Nucl. } & \multicolumn{3}{|c|}{$\begin{array}{l}\text { Energy } \\
{[\mathrm{MeV}]}\end{array}$} & Trans. & \multicolumn{2}{|c|}{$\begin{array}{l}\mathrm{B}\left(\mathrm{E} 1 ; \mathrm{I}_{\nu}^{\pi} \rightarrow \mathrm{J}_{\nu^{\prime}}^{\pi^{\prime}}\right)\left[10^{-3} \mathrm{e}^{2} \mathrm{fm}^{2}\right] \\
\mathrm{B}\left(\mathrm{E} 2 ; \mathrm{I}_{\nu}^{\pi} \rightarrow \mathrm{J}_{\nu^{\prime}}^{\pi^{\prime}}\right)\left[10^{4} \mathrm{e}^{2} \mathrm{fm}^{4}\right] \\
\mathrm{B}\left(\mathrm{E} 3 ; \mathrm{I}_{\nu}^{\pi} \rightarrow \mathrm{J}_{\nu^{\prime}}^{\pi^{\prime}}\right)\left[10^{6} \mathrm{e}^{2} \mathrm{fm}^{6}\right]\end{array}$} \\
\hline & $\mathrm{J}_{\nu^{\prime}}^{\pi^{\prime}}$ & Exp. & QPM & $\mathrm{E} \lambda$ & $\begin{array}{ll}\mathrm{I}_{\nu}^{\pi} & \text { Exp. } \\
\end{array}$ & QPM \\
\hline \multirow{4}{*}{${ }^{120} \mathrm{Sn}$} & $2_{1}^{+}$ & 1.171 & 1.171 & E2 & $0_{1}^{+} \quad 0.200(3)$ & 0.193 \\
\hline & & & & E1 & $3_{1}^{-} 2.02(17)$ & 1.82 \\
\hline & $3_{1}^{-}$ & 2.401 & 2.424 & E3 & $0_{1}^{+} \quad 0.115(15)$ & 0.110 \\
\hline & $1_{1}^{-}$ & 3.279 & 3.203 & E1 & $0_{1}^{+} 7.60(51)$ & 7.6 \\
\hline \multirow[t]{4}{*}{${ }^{122} \mathrm{Sn}$} & $2_{1}^{+}$ & 1.141 & 1.137 & $\mathrm{E} 2$ & $0_{1}^{+} \quad 0.194(11)$ & 0.190 \\
\hline & & & & E1 & $3_{1}^{-} 2.24(14)$ & 2.06 \\
\hline & $3_{1}^{-}$ & 2.493 & 2.486 & E3 & $0_{1}^{+} \quad 0.092(10)$ & 0.099 \\
\hline & $1_{1}^{-}$ & 3.359 & 3.281 & E1 & $0_{1}^{+} 7.16(54)$ & 7.02 \\
\hline \multirow[t]{4}{*}{${ }^{124} \mathrm{Sn}$} & $2_{1}^{+}$ & 1.132 & 1.133 & $\mathrm{E} 2$ & $0_{1}^{+} \quad 0.166(4)$ & 0.174 \\
\hline & & & & E1 & $3_{1}^{-} 2.02(16)$ & 1.98 \\
\hline & $3_{1}^{-}$ & 2.614 & 2.645 & E3 & $0_{1}^{+} \quad 0.073(10)$ & 0.087 \\
\hline & $1_{1}^{-}$ & 3.490 & 3.549 & E1 & $0_{1}^{+} \quad 6.08(66)$ & 6.27 \\
\hline \multirow[t]{4}{*}{${ }^{126} \mathrm{Sn}$} & $2_{1}^{+}$ & 1.141 & 1.151 & $\mathrm{E} 2$ & $0_{1}^{+}-$ & 0.140 \\
\hline & & & & E1 & $3_{1}^{-}-$ & 1.74 \\
\hline & $3_{1}^{-}$ & 2.720 & 2.792 & E3 & $0_{1}^{+}-$ & 0.079 \\
\hline & $1_{1}^{-}$ & - & 3.856 & E1 & $0_{1}^{+}-$ & 5.8 \\
\hline \multirow[t]{4}{*}{${ }^{128} \mathrm{Sn}$} & $2_{1}^{+}$ & 1.168 & 1.154 & $\mathrm{E} 2$ & $0_{1}^{+}-$ & 0.097 \\
\hline & & & & E1 & $3_{1}^{-}-$ & 1.07 \\
\hline & $3_{1}^{-}$ & - & 2.849 & E3 & $0_{1}^{+}-$ & 0.081 \\
\hline & $1_{1}^{-}$ & - & 4.115 & E1 & $0_{1}^{+}-$ & 5.56 \\
\hline \multirow[t]{4}{*}{${ }^{130} \mathrm{Sn}$} & $2_{1}^{+}$ & 1.221 & 1.204 & $\mathrm{E} 2$ & $0_{1}^{+}-$ & 0.066 \\
\hline & & & & E1 & $3_{1}^{-}-$ & 1.11 \\
\hline & $3_{1}^{-}$ & - & 2.861 & E3 & $0_{1}^{+}-$ & 0.098 \\
\hline & $1_{1}^{-}$ & - & 4.094 & E1 & $0_{1}^{+}-$ & 5.53 \\
\hline
\end{tabular}

energies and transition probabilities of these states are presented in Table I and compared to the known experimental data. The well reproduced properties of the $2_{1}^{+}$ and $3_{1}^{-}$states are important for a trustable description of the two-phonon quadrupole-octupole $\mathrm{J}^{\pi}=1_{1}^{-}$state which is of our particular interest. The experimental data on excitation energies and transition strengths 20], available for ${ }^{120 \div 130} \mathrm{Sn}$, are well described by the QPM as seen from Table I.

The QPM predicts for the lowest-lying $1^{-}$states excitation energies varying from $\mathrm{E}_{x}=3.203 \mathrm{MeV}$ to $\mathrm{E}_{x}=4.115 \mathrm{MeV}$ in ${ }^{120 \div 130} \mathrm{Sn}$ (see Table I). In these isotopes the $1_{1}^{-}$states are at a level of about $90 \%$ predominantly given by two-phonon quadrupole-octupole configurations. Since with increasing neutron number the energy of the latter becomes larger, certain other, higherlying, two-phonon configurations also start to contribute. The three-phonon configurations are most important for the lower-mass $\mathrm{Sn}$ isotopes because of their open shell structure. The reduction correlates with the decrease of collectivity when approaching the $\mathrm{N}=82$ shell closure. The QPM results for the boson forbidden E1 transitions 9] from the two-phonon $1^{-}$state to the ground state and between the $3_{1}^{-}$and $2_{1}^{+}$states are also shown in Table $\llbracket$ The experimental data available for ${ }^{116} \mathrm{Sn} \div{ }^{124} \mathrm{Sn}$ [7] are well described.

Of central interest for this work are the $1^{-}$states above the two-phonon dipole state and below the neutron threshold and their evolution with the neutron excess. These states are eventually to be identified as PDR modes related to excitations of the neutron skin as found in ${ }^{208} \mathrm{~Pb}[3]$. In fact, in all nuclei considered here the first three $1^{-}$QRPA states contribute to this energy region. They are rather well separated by a energy gap of more than $1.3 \mathrm{MeV}$ from other higher-lying one-phonon $1^{-}$ states which are more likely to belong to the low-energy tail of the GDR. QRPA and QPM results on the total PDR strengths and average centroid energies (defined by the ratios of energy and non-energy weighted sum rules) in Sn isotopes are compared in Tab! As a general result we find a correlation of the total PDR strength and the neutron excess as reflected by the increase of the total PDR strength with mass number. The PDR centroid energies, however, decrease with mass number. They are only weakly affected by multi-phonon admixtures.

The calculated energies and transition strengths are in a good agreement with experimental data 10]. The detailed analysis of the E1 strength distribution over the $1^{-}$excited states in ${ }^{120 \div 130} \mathrm{Sn}$ isotopes reveals that the major part of the strength is concentrated in the states with relatively large one-phonon $\left[1_{2}^{-}\right]$or $\left[1_{3}^{-}\right]$components. For the total PDR strengths the differences between the QRPA and QPM are significant (see Table III). Although the contribution of the higher-lying one-phonon components to the wave function structure of the low-lying $1^{-}$ states is small their influence on the total E1 transition is important because of partial admixtures of the large collective one-phonon matrix elements from the GDR region. The QPM B(E1) values are enhanced significantly by factors ranging from about 2 to 1.5 for $A=120$ to $A=130$, respectively. Thus, the PDR transition strengths contain a considerable fraction of strength from higher-lying (GDR) states, virtually admixed through the anharmonic QPM interactions. These admixtures, in fact, overcompensate the loss of ground state transition strength resulting from the dissipation of the one-phonon states into the multi-phonon components. In ${ }^{122} \mathrm{Sn}$ and ${ }^{130} \mathrm{Sn}$ the QPM calculations exhaust about $85 \%$ of the PDR one-phonon strength in the bound energy region. In Table II also the relative contribution of the PDR to the Energy Weighted Sum Rule (EWSR) is given.

An interesting question is to relate the PDR modes to the neutron skin. While in [3] this was done on the level of transition densities here we analyze the PDR strength distributions as functions of $\delta r$, eq. [4 We find two well separated regions with a sudden increase in the slope beyond $A=126$ where $\delta r$ starts to exceed $0.2 \mathrm{fm}$. Hence, a clear signal on the thickness of the neutron skin is obtained allowing to extract information on nuclear shapes from PDR data.

The QRPA and QPM dipole strength distributions be- 
TABLE II: Reduced transition probabilities and mean energies of the PDR modes in ${ }^{120} \mathrm{Sn} \div{ }^{132} \mathrm{Sn}$ calculated within QRPA are presented. The contribution of the PDR mode in the Energy Weighted Sum Rule (EWSR) is given in $\%$. For ${ }^{124}$ Sn the calculations are compared with the experimental data

\begin{tabular}{|c|c|c|c|c|c|c|c|}
\hline \multirow[t]{2}{*}{ Nucleus } & \multicolumn{3}{|c|}{$\langle E\rangle[\mathrm{MeV}]$} & \multicolumn{3}{|c|}{$\sum B(E 1) \uparrow\left[e^{2} f m^{2}\right]$} & \multirow[t]{2}{*}{ EWSR (\%) } \\
\hline & Exp. & QRPA & $\overline{\mathrm{QPM}}$ & Exp. & QRPA & $\overline{\mathrm{QPM}}$ & \\
\hline${ }^{120} \mathrm{Sn}$ & - & 6.839 & 6.920 & - & 0.142 & 0.289 & .185 \\
\hline${ }^{122} \mathrm{Sn}$ & - & 6.695 & 6.708 & - & 0.181 & 0.347 & .228 \\
\hline${ }^{124} \mathrm{Sn}$ & $6.5^{[10]}$ & 6.544 & 6.641 & $0.345(43)^{[10]}$ & 0.219 & 0.398 & .267 \\
\hline${ }^{126} \mathrm{Sn}$ & - & 6.330 & 6.442 & - & 0.261 & 0.416 & .304 \\
\hline${ }^{128} \mathrm{Sn}$ & - & 6.100 & 6.210 & - & 0.281 & 0.434 & .313 \\
\hline${ }^{130} \mathrm{Sn}$ & - & 5.940 & 6.150 & - & 0.337 & 0.495 & .361 \\
\hline${ }^{132} \mathrm{Sn}$ & - & 5.900 & - & - & 0.360 & - & .380 \\
\hline
\end{tabular}

low neutron threshold in ${ }^{122} \mathrm{Sn}$ and ${ }^{130} \mathrm{Sn}$ are compared in Figl2 The lowest QPM 1- ${ }^{-}$states do not have QRPA counterparts because they are the quadrupole-octupole two-phonon states. The QPM anharmonicities introduce a considerable fragmentation - in these cases up to about 80 states. Compared to [10] we find less fragmentation because our two- and three-phonon configuration spaces are somewhat smaller. However, the obtained for PDR total strength and centroid energy is in very reasonable agreement with the available experimental data in ${ }^{124} \mathrm{Sn}$ (see Table II).

The lowest QRPA $1^{-}$states are mainly given by pure neutron two-quasiparticle excitations with small admixtures of higher-lying two-quasiparticle neutron and proton configurations on a level of a few percent or less. The dominance of neutron excitations in this energy region is in agreement with the results of previous PDR studies in Sn and other nuclei by relativistic random-phase approximation (RRPA) 21], DFT [22] and QPM [3]. The remarkable stability of the wave function structure over the isotopic chain resembles what is found theoretically also for the GDR. However, we emphasize that the PDR states are of a much stronger isoscalar (or eventually mixed isospin) content than the GDR, see also [3]. Hence, the PDR states cannot be considered as simply being the low-energy tail of the isovector GDR, despite the anharmonic admixtures discussed above. Rather, these dipole modes are of a genuine character which cannot be deduced by extrapolations from the GDR region. This conclusion is supported by the differences in transition densities and velocity fields discussed in [3].

In summary, neutron rich tin isotopes were studied in a semi-phenomenological approach combining HFB and QPM theory. The results confirm the success of such a microscopically inspired description. An important step in understanding the dipole spectra is to disentangle the PDR states from the low-energy two-phonon dipole states, achieved here by using the QPM approach with up to three-phonon configurations. For ${ }^{120} \mathrm{Sn} \div{ }^{132} \mathrm{Sn}$ we obtained low-energy dipole strength concentrated in a narrow energy interval such that a pygmy dipole resonance (PDR) can be identified. The evolution of the
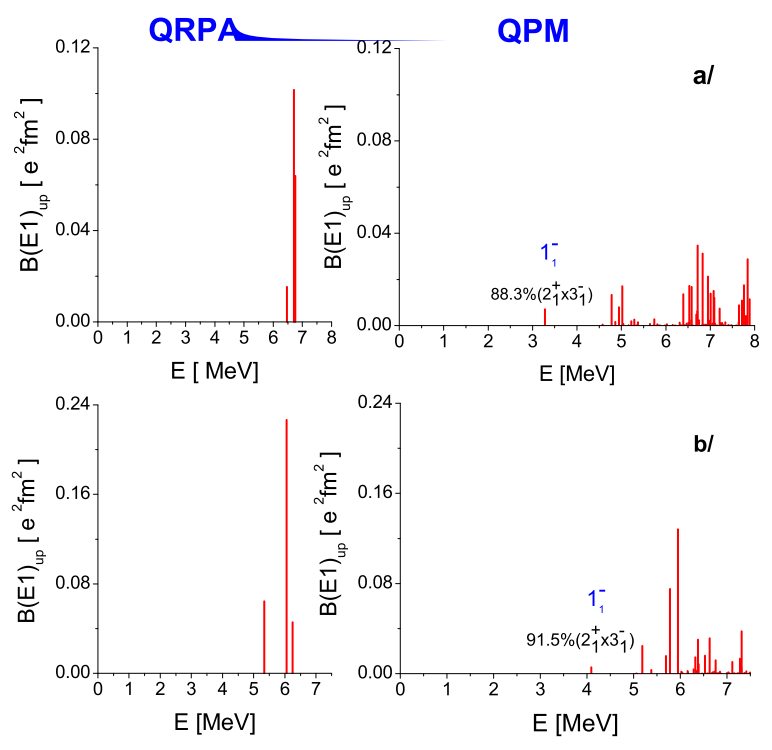

FIG. 2: QRPA and QPM calculations of the E1 strength distribution in a/ ${ }^{122} \mathrm{Sn}$ and $\mathrm{b} /{ }^{130} \mathrm{Sn}$ below the neutron threshold.

PDR strength distribution with neutron excess shows that transition strengths and energy locations are indeed closely correlated with the neutron skin. Hence, measurements of PDR strength distributions will provide information on nuclear shapes far off stability.

This work is supported by DFG, contract Le439/5 and Bulgarian Foundation for Scientific Research, contract $\mathrm{Ph} 1311$. 
[3] N. Ryezayeva al., Phys. Rev. Lett. 89, 272502 (2002).

[4] E. Tryggestad et al., Phys. Rev. C 67, 064309 (2003).

[5] A. Leistenschneider et al., Phys. Rev. Lett. 86, 5442 (2001).

[6] V.G. Soloviev, Theory of complex nuclei (Oxford: Pergamon Press, 1976).

[7] J. Bryssinck et al., Phys. Rev. C59 1930 (1999).

[8] M. Grinberg et al., Nucl. Phys. A573, 231 (1994).

[9] V.Yu. Ponomarev et al., Nucl. Phys. A635, 470 (1998).

[10] K. Govaert et al., Phys. Rev. C57, 2229 (1998).

[11] A. I. Vdovin et al., Sov. J. Part. Nucl. 14, 237 (1983).

[12] V. V. Voronov et al., Sov. J. Part. Nucl. 14, 1380 (1983).

[13] W. Kohn, L.J. Sham, Phys. Rev. 140, A1133 (1965).

[14] H. Lenske et al., Rep. Prog. Nucl. Part. Phys. 46, 187
(2001).

[15] F. Hofmann and H. Lenske, Phys. Rev. C57, 2281 (1998).

[16] F. Hofmann et al., Phys. Rev. C64, 034314 (2001).

[17] G.Audi, A.H. Wapstra, Nucl. Phys. A595, 409 (1995).

[18] A. Krasznahorkay et al., Phys. Rev. Lett. 82, 3216 (1999).

[19] A. Krasznahorkay et al., INPC, ed. by E. Norman et al., p.751 (2001).

[20] S. Raman et al., At. Data Nucl. Data Tabl. 78 (2001); T. Kibédi et al., At. Data Nucl. Data Tabl. 80 (2002).

[21] N. Paar et al., Phys. Rev. C67, 034312 (2003).

[22] J. Chambers et al., Phys. Rev. C50, R2671 (1994). 